\title{
Electrophysiological modulations of lexical prediction by conversation requirements
}

\author{
Cristina Baus ${ }^{1,2}$, A.-Sophie Dubarry ${ }^{3} \&$ F.-Xavier Alario ${ }^{1}$
}

1. Aix-Marseille Université, CNRS, LPC UMR 7290, 13331 Marseille, France

2. Universitat Pompeu Fabra, Center for Brain and Cognition, 08018 Barcelona, Spain

3. Aix-Marseille Université, CNRS, LPL UMR 7309, 13100 Aix-en-Provence, France

Word count:

Keywords: lexical prediction, production-based models, N400, social context

Address for correspondence:

Cristina Baus

Universitat Pompeu Fabra

Ramón Trias Fargas, 25-27, 08005

Barcelona 
Running head: Lexical prediction in conversation

\section{ABSTRACT}

Language mediates most of our social life and yet, despite such social relevance and ubiquity, little is known about language processing during social interactions. To explore this issue, two experiments were designed to isolate two basic components of a conversation: 1) the interplay between language production and comprehension systems, and 2) the participation of a social partner. We explored how prediction processes in language comprehension are modulated by two basic components of a conversation. Participants were asked to perform a cross-modal priming paradigm in two blocks, one involving only comprehension trials and another in which trials requiring production and comprehension were intermixed. In the first experiment, participants were alone during the task and in the second experiment, participants believed they were performing the task jointly with an interactive partner. A critical electrophysiological signature of lexical prediction was observed, the N400 component, allowing to assess its modulation across conditions and experiments. when production was involved in the task, the effect of lexical predictability was enhanced at the early stages of language comprehension (anticipatory phase), irrespective of the social context. In contrast, language production reduced the effect of lexical predictability at later stages (integration phase), only when participants performed the task alone but not in the social context. These results support production based-models and reveal the importance of exploring language considering its interactive nature. 
Running head: Lexical prediction in conversation

\section{INTRODUCTION}

The communicative success of any conversation requires the fine coordination of a complex set of processes, linguistic and non-linguistic, between two or more people (Garrod \& Pickering, 2004). These processes allow interlocutors to successfully alternate between the roles of speaker and listener, with exceedingly short gaps between turns (200 ms on average; Ruiter, Mitterer, \& Enfield, 2006; Stivers et al., 2009), while, at the same time, keeping track and integrating the information content being exchanged (e.g., Magyari \& de Ruiter, 2012). The fact that language unfolds so efficiently in conversation suggests that individuals are very skilled at talking to each other.

The simplicity with which we converse with others in our daily life contrasts with the difficulty of studying language processing in dialogue. Consequently, the study of language and its neurobiology comes mainly from studies exploring language production and comprehension separately and within the confines of individual brains. Thus, questions such as how we engage so easily in conversations, or how language processes unfold during a conversation (relative to a monologue) have remained elusive. The present study aimed to advance our understanding of the basic mechanisms of language in conversation by focusing on two computational components arguably central to any conversation, a) the fact that conversing requires interleaving production and comprehension processes, and b) the fact that conversing requires the interaction with a real interlocutor. We describe these two components in detail.

1.1. The interplay between language production and comprehension processes.

A number of recent theoretical proposals describe the language processing architecture as a unified system in which the interaction between production and 
Running head: Lexical prediction in conversation

comprehension is emphasized. Within these frameworks, the core concept of “prediction processes” has been identified as a shared mechanism at the service of both language production and comprehension (e.g. in prediction-through-production models; (Dell \& Chang, 2013; Pickering \& Garrod, 2007; Pickering et al., 2013). Language comprehension is thought to involve the pre-activation of linguistic information (e.g. lexical predictions), and this is accomplished through the same mechanisms the speaker uses to produce language. In other words, the same cognitive (and often neurophysiological) mechanisms have been hypothesized to underlie processes of speech production and prediction for comprehension (Huettig, 2015; Molinaro, Monsalve, \& Lizarazu, 2016; Pickering \& Garrod, 2007).

Several studies have reported evidence that highlights the links between production and prediction during language comprehension (Borovsky, Elman, \& Fernald, 2012; Drake \& Corley, 2015; Ito, Corley, Pickering, Martin, \& Nieuwland, 2016; Mani, Daum, \& Huettig, 2016; Mani \& Huettig, 2012). Of highest importance here are those studies showing a direct influence of production on prediction processes during comprehension. Martin, Branzi and Bar (2018) reported electrophysiological evidence that lexical prediction in reading is affected by the availability of the speech production system. Participants were asked to read sentences with predictable or unpredictable ends, while at the same time they had to perform a secondary production task involving speech or not (phoneme production vs. tongue movements). Predictability effects, indexed by the ERP component N400, which is larger for unpredictable than predictable endings, were reduced when the secondary task involved speech. In apparent contrast, Hintz, Meyer and Huettig, (2016) reported a larger effect of predictability (indexed by faster reading times), when speech production was intermixed with comprehension in the task. Faster reading times for predictable versus 
Running head: Lexical prediction in conversation

unpredictable word-endings were observed when comprehension trials (sentence selfpaced reading) were intermixed with production trials (picture naming task), but not when the task only involved comprehension. Both findings suggest that predictability effects in language comprehension are modulated by the involvement of the language production system in the task. Whether lexical prediction is hampered or supported by the language production requirement could be determined by the interplay of production and comprehension processes (intermixed vs simultaneous) during the task.

Ito, Dunn and Pickering (2017) suggested that the involvement of production in prediction processes may vary in the course of language comprehension. Two core phases have been distinguished in the course of language comprehension: an anticipatory phase (the time before the onset of the target word) and an integration phase (the time after the onset of the target word; e.g., Dikker \& Pylkkänen, 2013; Dikker, Silbert, Hasson, \& Zevin, 2014; Nieuwland et al., 2019). In Ito et al.’s experiment (2017), participants were asked to listen to sentences (e.g., "the man was gathering honey when he was stung by a BEE/CAT and gave a cry”) or to shadow the same sentences (repeat the utterance back while listening to it). Concurrently, they were viewing objects representing the predictable $(B E E)$ or the unpredictable $(C A T)$ word. Predictability effects, indexed by looking times at the objects, were modulated by the involvement of speech production in the task, and their direction varied in the course of comprehension. In the anticipation phase (i.e. the time before the predictable word was presented), eye fixations to the target-word picture occurred earlier when participants were shadowing (i.e., listening and speaking) than when they were just listening. Conversely, in the integration phase (i.e. the time after the predictable word was presented), fewer fixations to the target were observed in shadowing than in listening. These results were interpreted as an indication that the engagement of speech 
Running head: Lexical prediction in conversation

production enhances prediction during the anticipatory phase of language comprehension (e.g., through top-down processes) because of the similar nature of both processes. For the same reason, speech production interferes with integration in language comprehension because the mechanisms of one and other are different (roughly: top-down vs bottom-up process).

The modulations reported in these three studies indicate that language production is involved in lexical prediction during comprehension. However, how and when this happens in the course of language comprehension remains largely unclear, owing to the diversity of the experiments and the different results that have been reported. Summarizing, during integration (processing of the target word), positive (Hintz et al., 2016), negative (Ito et al., 2017) and no effects (Martin et al., 2018) of production on lexical predictability have been reported. The present study investigated the influence of language production on lexical predictability focusing on its time course during language comprehension. To do so, participants performed a comprehension task which was, or was not, intermixed with a production task. Like in Martin et al. (2018), their EEG was recorded and the modulations of the N400 component, during the anticipation and integration phases, were taken as an index of lexical prediction during comprehension.

\subsection{The joint conversational context}

While most models describing the interplay between production and comprehension are focused on dialogues (e.g., Pickering \& Garrod, 2013), experimental research has largely ignored the contexts in which two or more interlocutors are linguistically involved (i.e., social interactive contexts). It has not been until recent years that cognitive neuroscience has started considering the two-person approach (i.e., 2 person-neuroscience, 2PN; e.g., García \& Ibáñez, 2014) as a necessary 
Running head: Lexical prediction in conversation

step to fully understand language, focusing on naturalistic language communication, and relying on experiments that include linguistic exchanges among interlocutors (e.g., Baus et al., 2014; Gambi, Van de Cavey, \& Pickering, 2015; Kuhlen \& Abdel Rahman, 2017).

Speakers’ linguistic choices are influenced by those of interlocutors (Clark \& Carlson, 1982), and the same happens with listeners' linguistic processing. For example, Rueschemeyer, Gardner and Stoner (2015) showed that listeners' comprehension is affected by what information is accessible to their co-listeners (i.e., their partners in a listening task). Participants, along with a confederate, were asked to read sentences that were plausible, implausible, or whose plausibility depended on previous auditory information provided uniquely to the participant. Such contextdependent sentences (e.g., “the boy has gills”) were semantically plausible with the auditory context received by the participant (e.g., "In the boy's dream, he could breathe under water"), but implausible from the point of view of the confederate, who did not receive such information. Implausible sentences and context-dependent sentences elicited a similar N400 effect on the participant, despite the latter being appropriately contextualized. This so-called "social N400 effect", was taken as a reflection of the comprehender modelling the knowledge states of co-listeners, even when this affected their own performance (see also Jouravlev et al., 2019; Westley, Kohút, \& Rueschemeyer, 2017, for replication of the “social-N400”). Following this idea, in the present study we further explored how listeners' lexical predictions are modulated by the presence of an interlocutor, and how they differ from processing language in isolation. 
Running head: Lexical prediction in conversation

\subsection{The present experiments}

In two experiments, we explored lexical prediction in comprehension by assessing the impact of a) the involvement of language production in the task, and b) the interaction with an interlocutor.

To explore the first issue, our experiments comprised two blocks, one in which participants only had to comprehend (C-block), and another block in which language production and comprehension were interleaved (CP-block). We focused exclusively on comprehension trials to test how the task-level requirement of producing language influenced lexical predictions by the listener. Lexical predictability was manipulated through color diagnosticity (e.g., Bramão et al., 2012; Tanaka \& Presnell, 1999), contrasting words that represent common objects with specific colors associated to them (SUN) and words having unspecific colors (UMBRELLA). It is agreed that processing words denoting color-related objects involves access to the lexical representation of color (Léger \& Chauvet, 2015; Yee, Ahmed, \& Thompson-Schill, 2012), which allowed to explore lexical predictability associated to color. In addition, we explored the two time phases in the course of language comprehension discussed above (Dikker et al., 2014; Ito et al., 2017; Molinaro et al., 2016), namely the anticipatory and integration phases. To do so, a cross-modal priming paradigm was used with visual primes and auditory targets. There were 2 types of visual word primes, predictable or unpredictable regarding color (sun/umbrella) and 3 types of auditory targets: color congruent (sun-YELLOW) or incongruent with the color predictable word (sun-BLUE) and color unpredictable (but congruent) word (umbrella-YELLOW). The EEG signatures of lexical predictability were indexed by the N400. 
Running head: Lexical prediction in conversation

The N400 components is one of the most studied ERP components in the field of language comprehension (Kutas \& Federmeier, 2011, for a review) and therefore very well described in terms of timing (300-500ms after target word presentation), topography (centro-posterior) and functionality (e.g., lexico-semantic processing). Importantly here, the N400 effect has been taken as direct evidence of prediction during language comprehension (e.g., Lau, Holcomb, \& Kuperberg, 2013). Regarding color diagnostic words, larger N400 amplitudes have been reported for non-color diagnostic objects than for color diagnostic ones (Bramão et al., 2012; Redmann, FitzPatrick, Hellwig, \& Indefrey, 2014). Thus, focusing on the electrophysiological component N400 allowed us to test what aspects of language interaction impact on lexical prediction during real-time language processing. By exploring pre-stimulus and poststimulus brain activity we were able to determine the effect of speech production during the anticipatory and integration phases of language comprehension.

To explore the impact of the social context, predictability effects were tested in two Experiments: the solo context (Experiment 1), where participants were alone doing the task, and the joint context (Experiment 2), where participants believed they were performing the task jointly with a partner. Differences between experiments regarding lexical predictability were informative of the influence of a real interactive-partner on language processing.

\section{Experiment 1. Lexical predictability in a solo context}

\subsection{METHOD}

\subsubsection{Participants}

Thirty French native speakers (mean age $=21$; S.D = 2.6; 23 females) from the XXX community took part in the experiment in exchange of monetary compensation 
Running head: Lexical prediction in conversation

or course credits. The study had received appropriate ethical approval (XXX). All participants gave their informed consent. They were right-handed and declared not having any neurological or hearing problem. Three participants were discarded after they declared having learning problems or epileptic episodes during infancy. In addition, four participants were excluded because of an excessive number of artifacts in the EEG signal, with more than $25 \%$ of trials affected (see below for details). Thus, the final pool of participants comprised 23 participants (mean age $=21$; S.D $=1.5 ; 17$ females).

\subsubsection{Materials}

The experiment consisted in a cross-modal priming task, with visual primes and auditory targets. The visual primes comprised 150 different words. One hundred of them were selected for their referent object having a color strongly associated to them (e.g., SUN; hereafter “color predictable”), while the remaining 50 did not have a specific color associated to them (e.g., UMBRELLA; hereafter “color unpredictable”). This imbalance was designed to afford a larger proportion of congruent than incongruent trials across the experiment (50\% predictive congruent trials; $25 \%$ predictive incongruent trials; 25\% non-predictive trials; see below).

To test the agreement on the color of the corresponding objects, a separate group of participants $(\mathrm{N}=26)$ was asked to determine the color to which each word's corresponding object was mostly associated. Color agreement with the color used in the experiment was $89 \%$ for color predictable words and $25 \%$ for the color unpredictable words. The two sets of words were matched for their lexical frequency, number of letters, number of syllables, orthographic neighbors, familiarity, imageability and AoA (all ts $<1$ ). The words were presented visually in a gray font color (RGB: 99,99,99) with a background color in lighter gray (RGB: 128,128,128). 
Running head: Lexical prediction in conversation

The auditory targets were eight different auditory color words translated from French as “black”, “white”, “red”, “blue”, “green”, “orange”, “yellow”, and "brown”. A female French speaker recorded different tokens of the color names to avoid repetition of the exact same acoustic token throughout the experiment.

\subsubsection{Design}

The experiment comprised two blocks: a comprehension block (C-block) in which participants were engaged in the cross-modal priming task (visual primes, auditory targets), and a comprehension/production block (CP-block), in which the cross-modal priming trials were intermixed with trials requiring the production of an overt response in Stroop-like task.

The visual primes and auditory targets were analyzed separately, and linked to the anticipatory and integration phases, respectively. The anticipatory phase was timelocked to the onset of the visual prime presentation; the integration phase was timelocked to the onset of the auditory target presentation.

In the anticipatory phase, word predictability was explored by comparing two prime-level conditions: color predictive words (“sun”) and color non-predictive words (“umbrella”). The 50 non-predictive words were presented only once in each of the Cand CP-blocks. Among the predictive prime words, 50 were presented only once (to be paired with congruent targets during integration). The other 50 predictive prime words were presented twice (to be paired with both congruent and incongruent targets across the resulting 100 trials).

In the integration phase, word predictability was explored by comparing activity between three target-level conditions: color predicted-congruent (sun-YELLOW), color predicted-incongruent (sun-BLUE), and color non-predicted (umbrella- 
Running head: Lexical prediction in conversation

YELLOW). The color predicted-congruent condition involved all the predictive prime words. The color predicted-incongruent condition involved half of the predictive prime words. The color non-predicted condition involved all of the non-predictive prime words. This imbalance was designed to ensure a larger proportion of congruent (50\%) than incongruent (25\%) or non-predictive (25\%) trials, to maintain the contextual of the color predictive value.

The design described above for the anticipatory and the integration phases applied equally to the C-block and to the CP-block. For the CP-block, in addition, filler trials with the prime printed in color were included to elicit the oral production of color names.

\subsubsection{Procedure}

As mentioned, the experiment was divided in two blocks: one with comprehension trials only (C-block) and another in which comprehension and production trials were intermixed (CP-block). Before each block, participants were informed whether they were in a C-block or in a CP-block. In the C-block, participants were explained that only comprehension trials (cross-modal priming) would be presented. Thus, no task was asked from them. In the CP-block, participants were explained that there would be comprehension trials and trials in which they would see a word and have to overtly produce the ink color (production trials). At the beginning of each trial, a visual cue (a circle or a square) was used to determine whether a given trial corresponded to comprehension or production. For half the participants, the circle was associated to comprehension and the square to production, while the reverse was true for the remaining participants. In the C-block, although uninformative, the cue used to indicate comprehension trials in the CP-block was also used. 
Running head: Lexical prediction in conversation

The sequence of events within a given trial was as follows. A fixation point (*) was presented for $500 \mathrm{~ms}$ followed by a blank interval of $300 \mathrm{~ms}$. Immediately after, the visual cue (square or circle) and the word prime (embedded on it) were presented for 500 ms followed by a masking sequence \#\#\#\#\#. To avoid an anticipation of the timing of target presentation, and to resemble as much as possible speaking trials (important for comparison to Experiment 2 purposes; see below), the sequence of \#\#\#\#\# remained on the screen for a random period between 450 and 800 ms, which was followed by the auditory presentation of the color word. The ITI was $1000 \mathrm{~ms}$ and a blank screen appeared allowing participants to blink if necessary. Before starting the experiment, participants received 6 familiarization trials whose data were not included in the analysis.

\subsubsection{EEG recordings and analysis}

Participants were tested in an EEG Faraday cage in order to reduce the $50 \mathrm{~Hz}$ electric line interference. The experimental paradigm was implemented and presented through the experimental software Presentation ${ }^{\circledR}$ (Version 20.3, Neurobehavioral Systems, Inc., Berkeley, CA). During the experiment, the electroencephalogram (EEG) was continuously recorded from 128 scalp electrodes located according to the standard 10-20 system positions, using Ag/AgCl active electrodes and CMS and DRL electrodes (Biosemi BV, Amsterdam). The sampling rate was set to $512 \mathrm{~Hz}$ (filters: DC to $104 \mathrm{~Hz}$, $3 \mathrm{~dB} /$ octave slope). Vertical (VEOG) and horizontal (HEOG) were recorded from 4 additional surface electrodes (Ag/AgCl) located above and next to the two outer canthi of both eyes. In addition, electrodes for offline reference were placed on the left and right mastoids. 
Running head: Lexical prediction in conversation

Offline analyses were conducted under Matlab (2016, MathWorks, Natick, MA), using the Brainstorm toolbox (Tadel, Baillet, Mosher, Pantazis, \& Leahy, 2011). Data were first bandpass filtered $(0.1-30 \mathrm{~Hz})$ and re-referenced to the average activity of the two mastoids. Independent component analysis (ICA, Infomax algorithm, 25 components) was performed on the continuous data to correct for blinks and ocular movements. A maximum of two components was removed from each participant. EEG activity exceeding $+/-100 \mu \mathrm{V}$ was considered artifact and therefore dismissed. In the anticipatory phase (visual primes) epochs were segmented from -100 to 500ms around visual prime onset; in the integration phase (auditory targets) epochs were segmented from -100 to $700 \mathrm{~ms}$ around auditory target onset presentation. Epochs were averaged for each condition separately and the mean baseline activity was subtracted (-100 to 0ms).

Based on the typical time-course of the N400 (Curran, Tucker, Kutas, \& Posner, 1993), we explored a 200-ms window of interest defined between 300 and 500ms after prime or target word onset, respectively, for anticipatory and integration phases.

Analysis: Two major analyses were performed to assess the effect of lexical predictability in the time-window of interest (300-500ms after the word onset). First, we ran an ANOVA for each electrode separately considering lexical predictability and block (comprehension, comprehension/production) as fixed factors. The robustness of the effect of predictability and its interaction with block was determined by the number of electrodes showing significance (False-Discovery-Rate corrected across electrodes considering main effects and interaction). When relevant, a restricted analysis was conducted over the electrodes showing significant interactions to further explore directions of the predictability effect. Second, the average activity of all electrodes (n 
Running head: Lexical prediction in conversation

$=128$ ) in the N400 time-range was submitted to an ANOVA with lexical predictability and block (comprehension, comprehension/production) as fixed factors.

\subsection{RESULTS}

Anticipatory phase. As can be seen in Figure 1, 106 out of 128 electrodes revealed a significant effect of block in the N400 time-range $\left(\mathrm{F}_{\mathrm{corr}}>7, \mathrm{p}_{\mathrm{corr}}<.02\right)^{1}$, indicating that visual primes elicited more positive waveforms in the CP-block than in the C-block. The effect of block was further validated by the ANOVA performed on the average activity of all electrodes $\left(\mathrm{F}(1,22)=28, \mathrm{p}<.001, \eta^{2}=.56\right)$. A main effect of predictability was present in 70 electrodes $\left(\mathrm{F}_{\text {corr }}>5\right.$, $\left.\mathrm{p}_{\text {corr }}<.02\right)$, with larger negativities for unpredictable color words (umbrella) than predictable ones (sun; ANOVA collapsed activity of 128 electrodes: $\left.\mathrm{F}(1,22)=9.9, \mathrm{p}<.01, \eta^{2}=.31\right)$.

A)

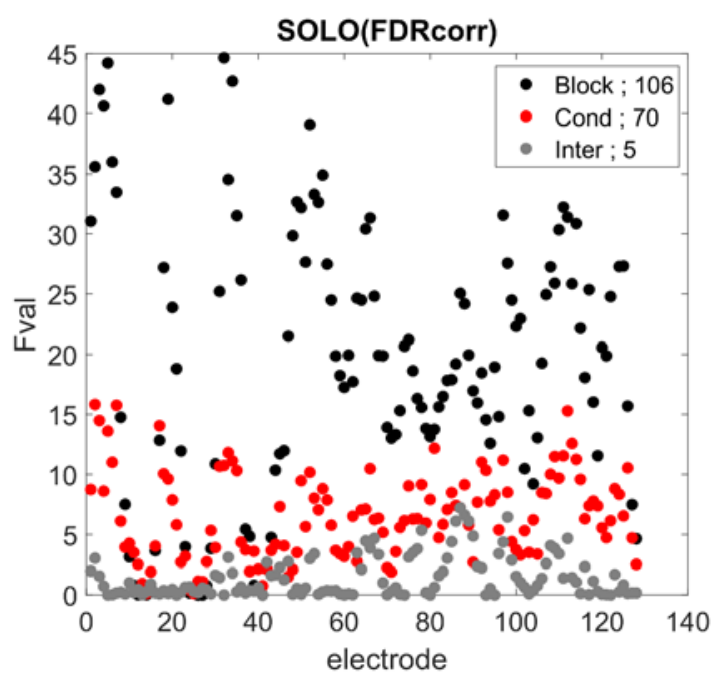

B)

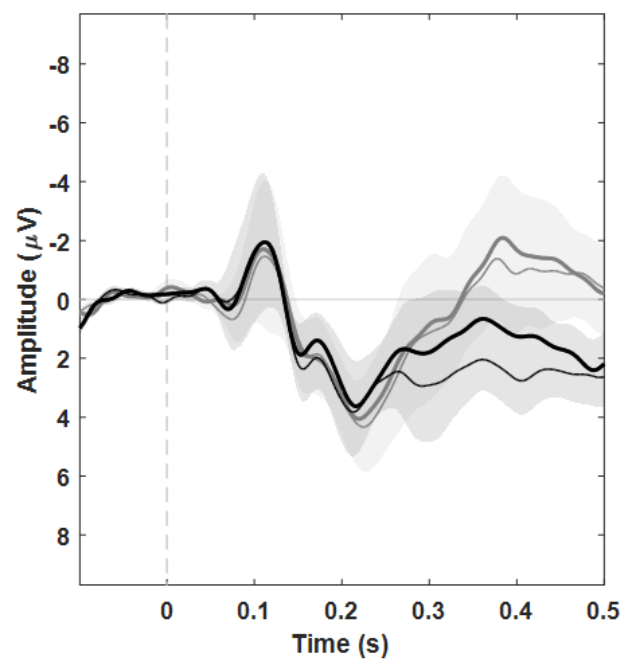

Figure 1. Figure A represents the statistical analysis (FDR corrected) in the anticipatory phase of the Solo Context (Experiment 1$)$ for each electrode $(n=128)$ considering the main effect of block (black dots), condition (red dots) and their interaction (gray dots). Figure B represents the grand average waveforms (collapsed for the 128 electrodes) for each block and condition. Gray lines represent the C-block and black lines represent the CP-block. Thick lines represent the unpredictable condition and thin lines the predictable condition. Shadow lines represent the standard error of the ERPs. 
Running head: Lexical prediction in conversation

Finally, the interaction between block and predictability was significant at five electrodes $\left(\mathrm{F}_{\text {corr }}>6\right.$, $\left.\mathrm{p}_{\mathrm{corr}}<.02\right)$, but it did not reach significance when average activity of the whole scalp electrodes was considered $(\mathrm{F}<1)$. The five electrodes showing a significant interaction (see Figure 2) were all located in the fronto-central region (e.g., FCz). Pairwise comparisons considering the average activity of these five electrodes revealed that the effect of predictability was significant in the CP-block $(\mathrm{t}(22)=3.1$, $\mathrm{p}<.01$ ), but not in the C-block $(\mathrm{t}<1)$. Thus, the involvement of production in the task resulted in an enhanced effect of lexical predictability in the anticipatory phase, and this was especially evident for electrodes at the fronto-central region.
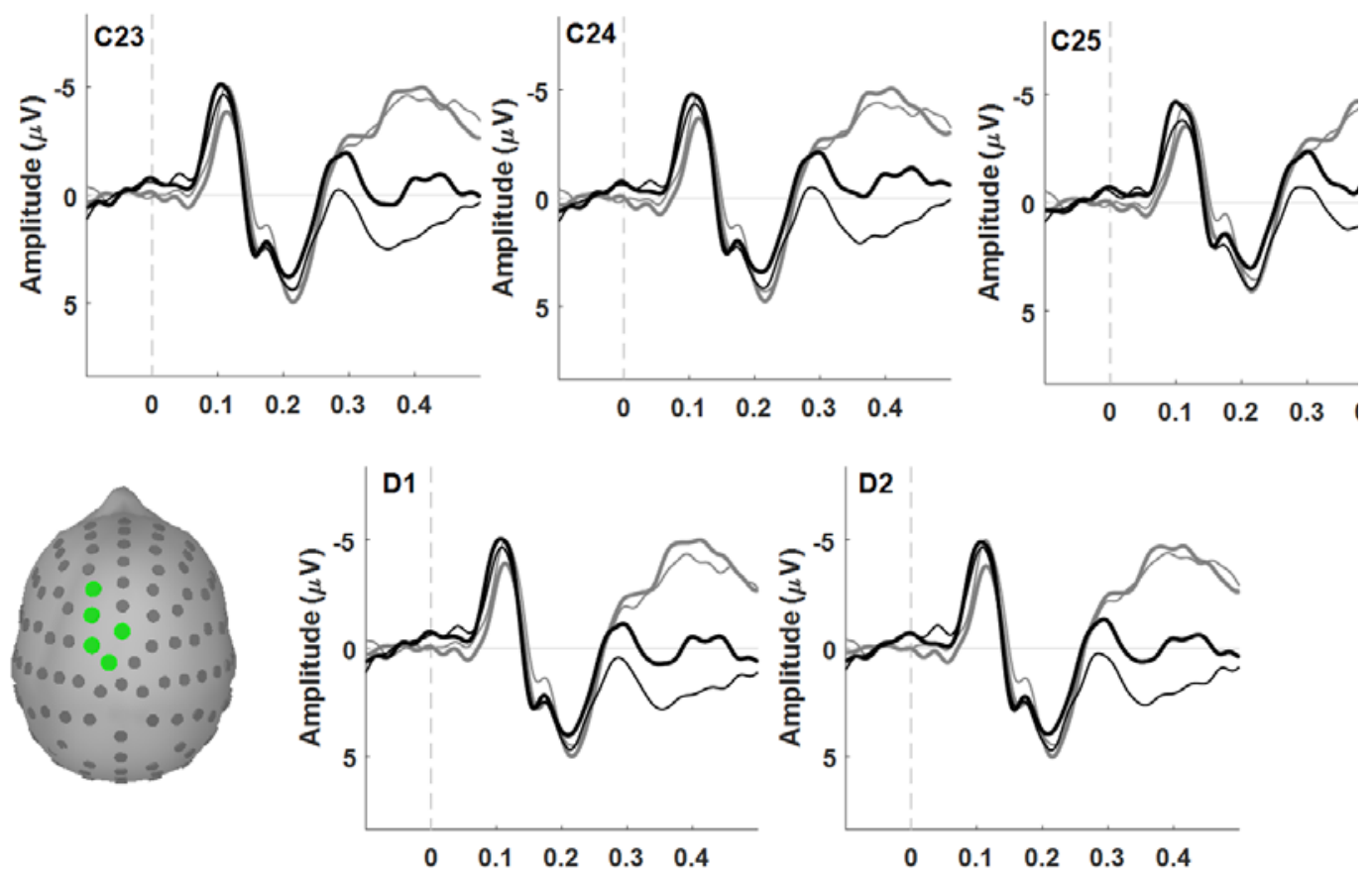

Figure 2. Grand average waveforms over the five electrodes showing a significant interaction between block and condition in the anticipatory phase of Experiment 1 (Solo context). Green dots in the head map indicate the position of the electrodes in the scalp. 
Running head: Lexical prediction in conversation

Integration phase. No differences were observed between blocks, neither at the electrode-level $\left(\mathrm{F}_{\mathrm{corr}}>7\right.$, $\left.\mathrm{p}_{\mathrm{corr}}<.0006\right)$, nor when the effect was explored considering the average activity of all electrodes ( $\mathrm{F}<1$; see Figure 3$)$. When considering the effect of predictability, 5 electrodes located over the central region (e.g., Cz) showed a significant effect $\left(\mathrm{F}_{\mathrm{corr}}>9\right.$, $\left.\mathrm{p}_{\mathrm{corr}}<.0006\right)$. This result was further validated in the analysis considering the average activity of all electrodes $\left(\mathrm{F}(2,44)=5.8, \mathrm{p}<.01, \eta^{2}=.21\right)$, showing that both incongruent and unpredictable conditions elicited a larger N400 than the congruent one, while no significant differences were observed between them (Incongruent-Congruent: $\mathrm{t}(22)=3.6, \mathrm{p}=.001$; Unpredictable-Congruent $: \mathrm{t}(22)=2.4$, $\mathrm{p}=.02$; Incongruent-Unpredictable: $\mathrm{t}<1$ ). The interaction between predictability and block was significant only when the average activity of all electrodes was considered $\left(\mathrm{F}(2,44)=3.5, \mathrm{p}<.05, \eta^{2}=.14\right)$, showing that predictability effects were modulated by block. Pairwise comparisons showed that in the C-block, the congruent condition differed from both the incongruent $(\mathrm{t}(22)=2.8, \mathrm{p}<.01)$ and the unpredictable conditions $(t(22)=4, p<.001)$, while no differences were observed between the incongruent and the unpredictable condition $(t<1)$. In the CP-block, the congruent condition differed from the incongruent $(\mathrm{t}(22)=2.2, \mathrm{p}<.05)$ but not from the unpredictable condition $(\mathrm{t}<1)$, and no differences were observed between the incongruent and unpredictable condition $(\mathrm{t}(22)=1.6, \mathrm{p}=.12)$

In sum, this experiment showed that the effect of lexical predictability is modulated by interleaving production and comprehension trials in the task, and that this modulation depends on the time-point of processing during comprehension. The effect of lexical predictability was enhanced by the involvement of production during the anticipatory phase. However, lexical predictability effects were not observed when language production was involved in the task. 
Running head: Lexical prediction in conversation

In the second experiment, we tested how the task-wide effect of production on lexical prediction is modulated by the involvement of an interlocutor in the task.

A)

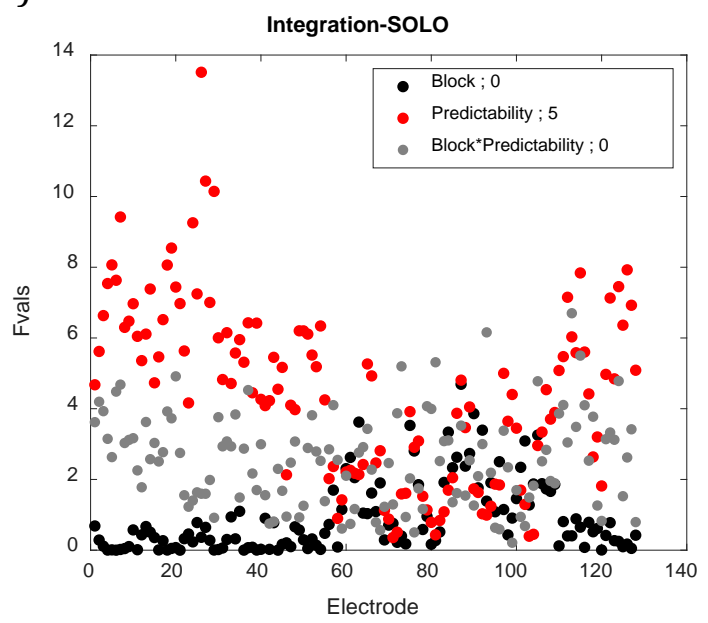

B)
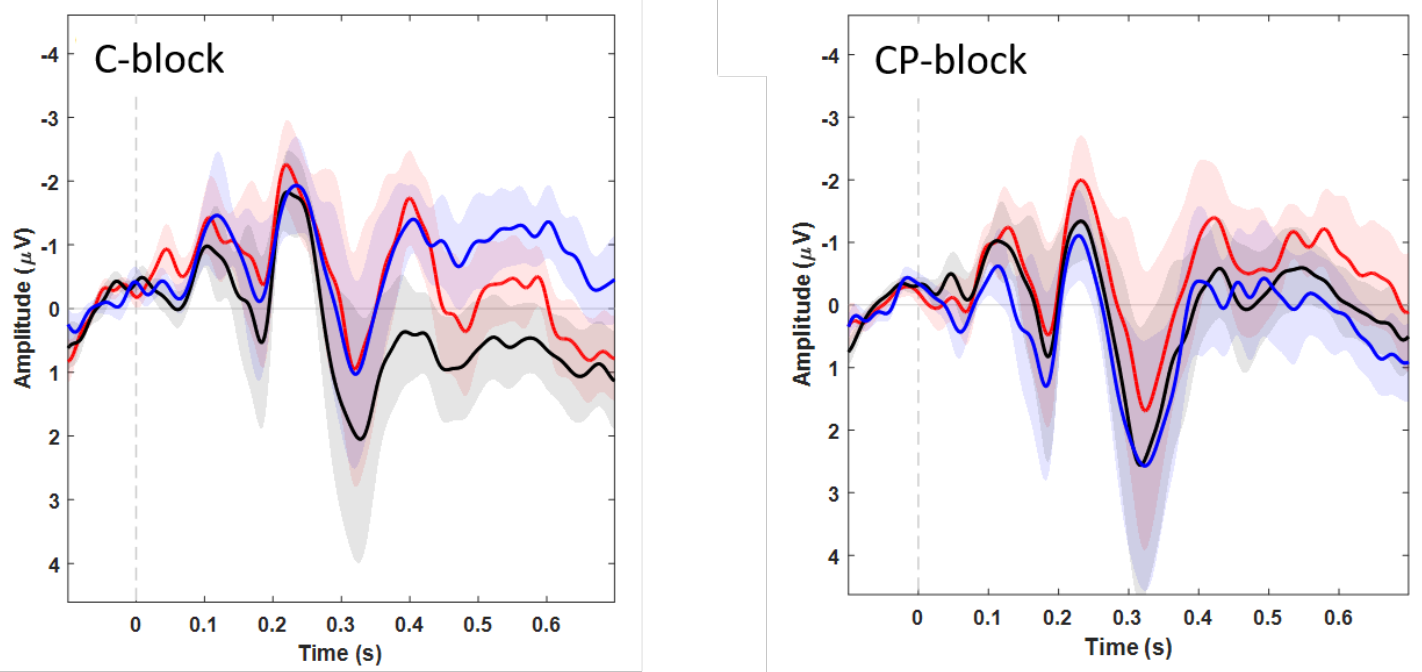

Figure 3. Figure A represents the statistical analysis (FDR corrected) in the integration phase of the Solo Context (Experiment 1$)$ for each electrode $(n=128)$ considering the main effect of block (black dots), condition (red dots) and their interaction (gray dots).

Figure B represents the grand average waveforms (collapsed for the 128 electrodes) for the Cblock (left panel) and the CP-block (right panel). Black lines represent the congruent condition, red lines the incongruent condition and blue lines the unpredictable condition. Shadow lines represent the standard error the ERPs. 
Running head: Lexical prediction in conversation

\section{Experiment 2. Lexical predictability in the social context.}

\subsection{METHODS}

\subsubsection{Participants}

Twenty-nine French native speakers (mean age = 19.7; S.D = 1.6; 23 females) from the same pool as those from Experiment 1 took part in this experiment. They were all right-handed and declared not having any neurological or hearing problems. One participant was excluded from the analysis after they declared having had epileptic absences during infancy. In addition, two participants were excluded because of excessive ERP artifacts (more that 25\% of the trials rejected). Thus, the final pool of participants was comprised of 26 participants (mean age $=19.5$; S.D $=1.6 ; 21$ females).

\subsubsection{Materials}

The same materials as in Experiment 1 were employed.

\subsubsection{Procedure}

The same procedure as in Experiment 1 was used, with one exception. Participants were induced to believe that they were participating with another person (confederate) performing the color priming task with them. At the beginning of the experiment, we introduced the confederate in person to the participant and explained the instructions to both of them at the same time, as if they would be performing the task jointly. The confederate was instructed to ask some clarification questions about the procedure, so the participant could be familiarized with the confederate's voice. Importantly, the confederate was the speaker that recorded the auditory color words that the participant would be hearing during the experiment. (In both experiments, we used the same auditory color words pre-recorded by the single confederate). Following the instructions, the participant was installed in the testing room and told that the other participant, the confederate, would be participating from another room. In truth, the 
Running head: Lexical prediction in conversation

confederate left the premises at that point. After the experiment, participants were debriefed about the experimental manipulation. They all declared believing another person, the confederate, was performing the experiment along with them. Thus, the only difference between experiments was that participants thought they were performing a joint task, when indeed they were as alone as in Experiment 1.

\subsubsection{EEG recordings and analysis}

The same analyses as in Experiment 1 were performed.

\subsection{RESULTS}

Anticipatory phase. As shown in Figure 4, the results were very similar to those reported in Experiment 1. Out of the 128 electrodes, 105 showed a significant effect of block $\left(\mathrm{F}_{\text {corr }}>6.6\right.$, $\left.\mathrm{p}_{\text {corr }}<.02\right)$, such that the CP-block elicited a larger positivity than the C-block (ANOVA considering average activity of all electrodes: $F(1,25)=33.6, \mathrm{p}<$ $\left.0.001, \eta^{2}=.57\right)$. A significant effect of predictability was present in 54 electrodes $\left(F_{\text {corr }}\right.$ $\left.>6, \mathrm{p}_{\mathrm{corr}}<.02\right)$ and also when considering the average activity of all electrodes $(\mathrm{F}(1,25)$ $=8.5, \mathrm{p}<.01, \eta^{2}=.25$ ), revealing a larger negativity for unpredictable than for predictable color words. The interaction between predictability and block was significant over 5 electrodes distributed in posterior and temporal regions $\left(F_{\text {corr }}>6.6\right.$, $\mathrm{p}_{\text {corr }}<.02$; e.g., Oz, TP8; ANOVA considering the average activity of the all electrodes $\left(\mathrm{F}(1,25)=3.5, \mathrm{p}=.03, \eta^{2}=.10\right)$. Pairwise comparisons considering the average activity of those five electrodes revealed a significant (see Figure 5) difference between predictable and unpredictable color words in the CP-block $(\mathrm{t}(25)=4, \mathrm{p}<.001)$ but not in the C-block $(\mathrm{t}<1)$. 
A)

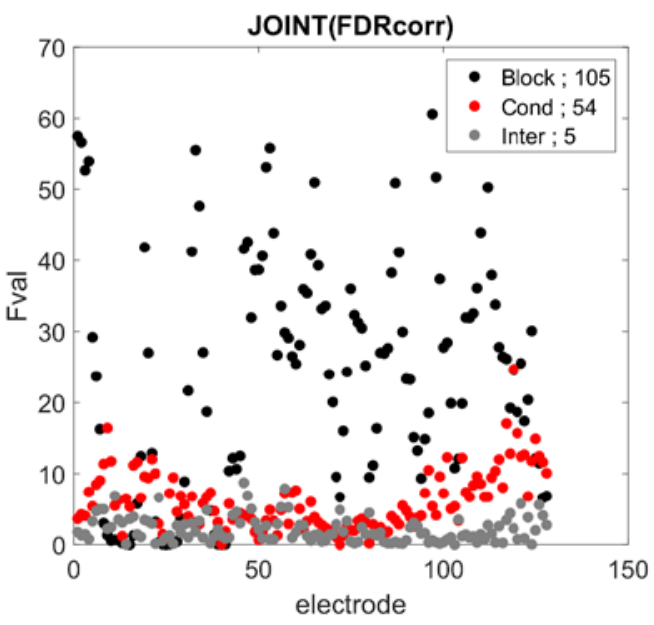

B)

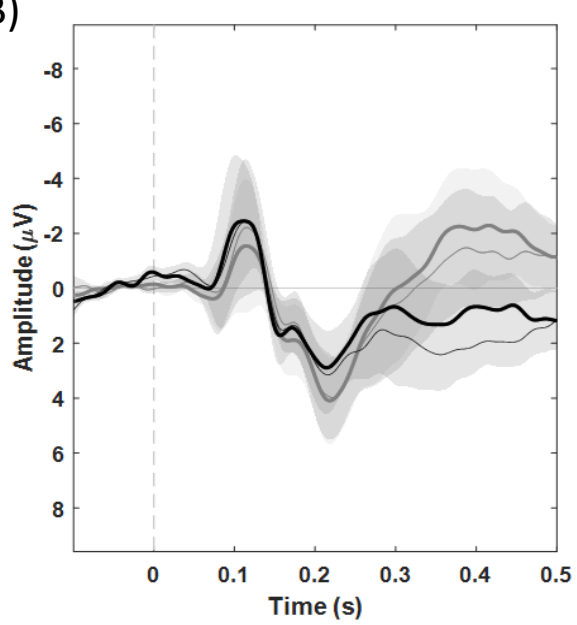

Figure 4. Figure A represents the statistical analysis (fdr corrected) in the anticipatory phase of the Joint Context (Experiment 2$)$ for each electrode $(n=128)$ considering the main effect of block (black dots), condition (red dots) and their interaction (gray dots). Figure B represents the grand average waveforms (collapsed for the 128 electrodes) for each block and condition. Gray lines represent the C-block and black lines represent the CP-block. Thick lines represent the unpredictable condition and thin lines the predictable condition. Shadow lines represent the standard error of the ERPs.

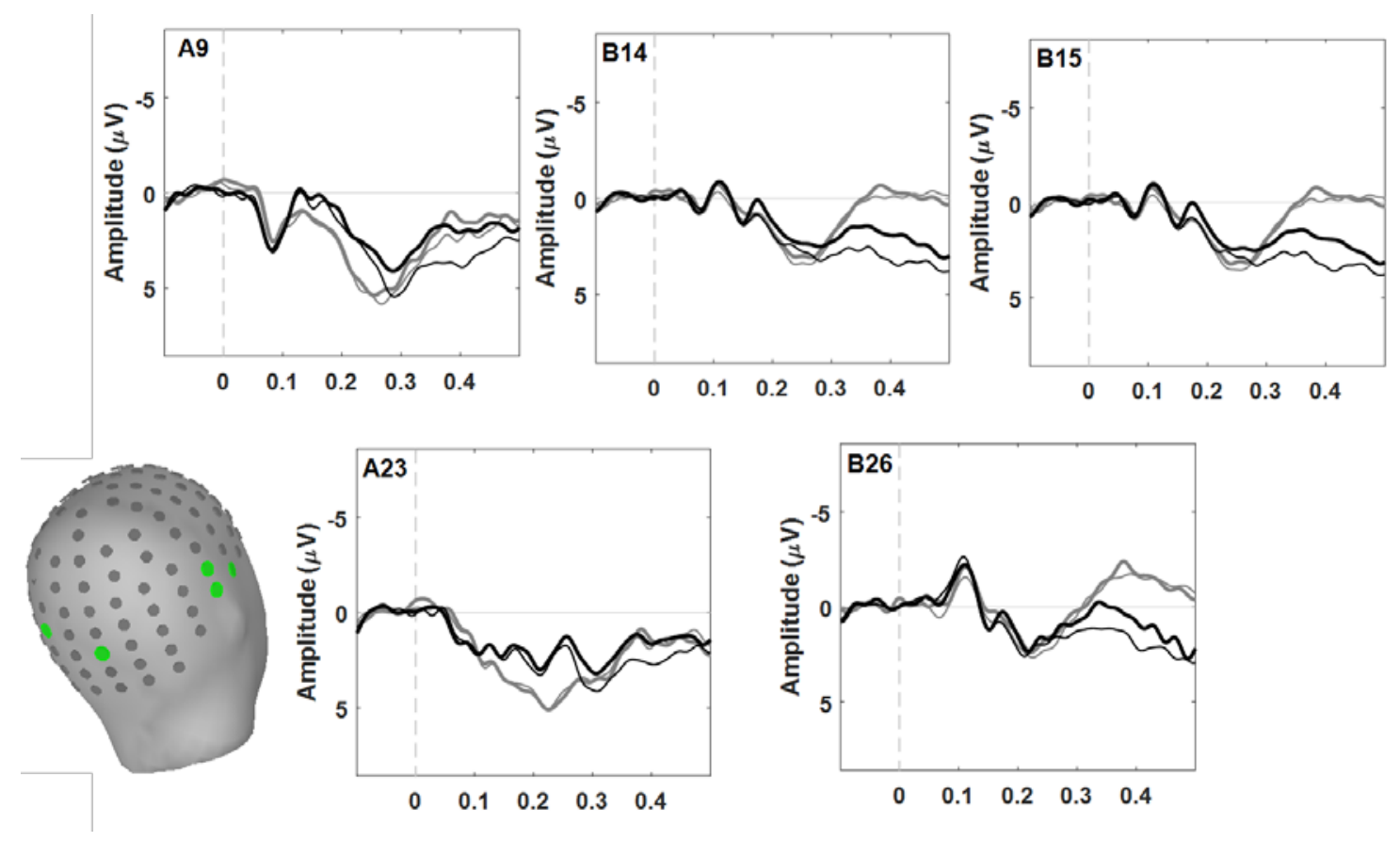

Figure 5. Grand average waveforms over the five electrodes showing a significant interaction between block and condition in the anticipatory phase (Joint context). Green dots in the head map indicate the position of the electrodes in the scalp. 
Running head: Lexical prediction in conversation

Thus, as in Experiment 1, the engagement of production in the task resulted in an enhancement of the lexical predictability effect. This effect however, was more prominent at posterior sites.

A)

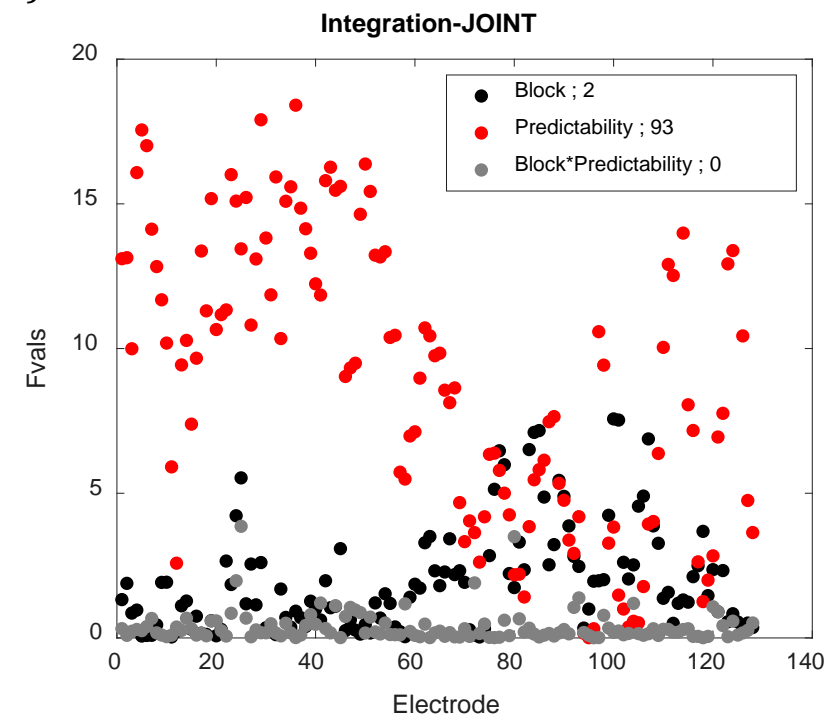

B)
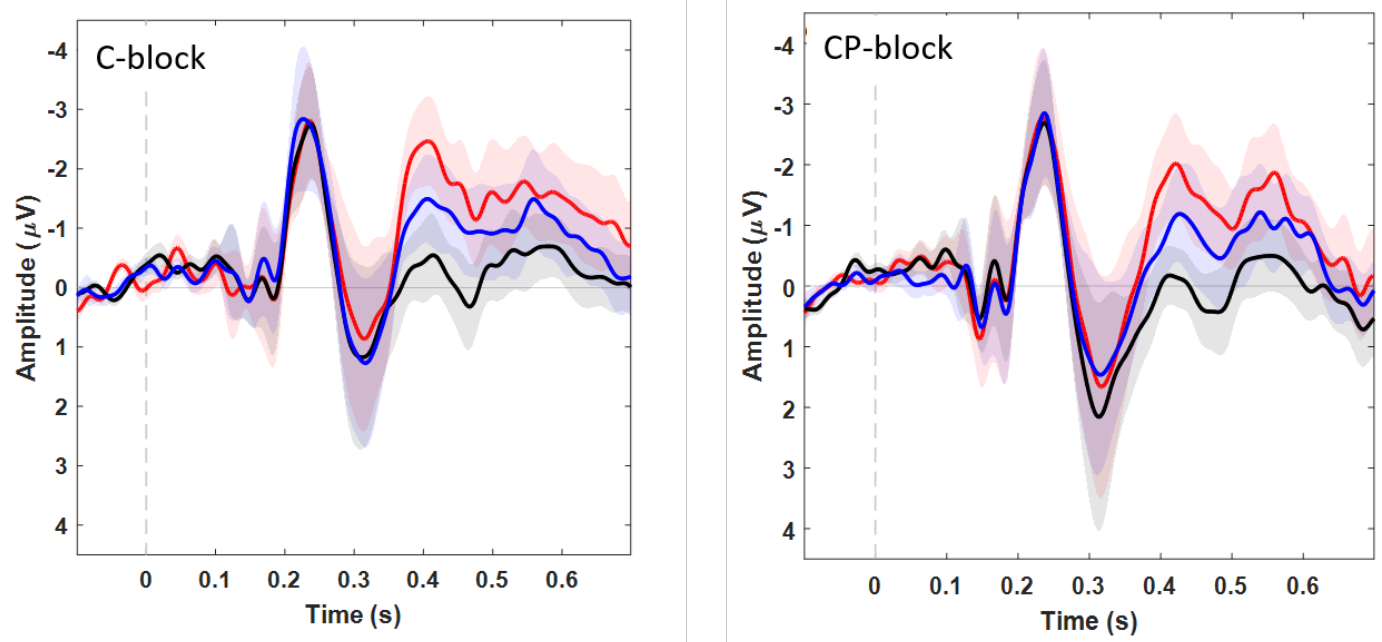

Figure 6. Figure A represents the statistical analysis (fdr corrected) in the integration phase of the Joint Context (Experiment 2$)$ for each electrode $(n=128)$ considering the main effect of block (black dots), condition (red dots) and their interaction (gray dots).

Figure B represents the grand average waveforms (collapsed for the 128 electrodes) for the Cblock (left panel) and the CP-block (right panel). Black lines represent the congruent condition, red lines the incongruent condition and blue lines the unpredictable condition. Shadow lines represent the standard error the ERPs. 
Running head: Lexical prediction in conversation

Integration phase: Two electrodes showed a significant difference between blocks $\left(\mathrm{F}_{\text {corr }}>7.5\right.$, $\mathrm{p}_{\text {corr }}<.01$; ANOVA considering average activity of all electrodes $\mathrm{F}(1,25)=3.2, \mathrm{p}=.08)$, with ERPs associated to the C-block eliciting a larger negativity than those associated to the CP-block. The effect of predictability was significant in 91 of the electrodes $\left(\mathrm{F}_{\mathrm{corr}}>7.5, \mathrm{p}_{\mathrm{corr}}<.01\right)$ and also when the average activity of all electrodes was considered $\left(\mathrm{F}(2,50)=13.4, \mathrm{p}<.001, \eta^{2}=.34\right)$, such as incongruent trials elicited a larger $\mathrm{N} 400$ than unpredictable $(\mathrm{t}(25)=3.4 ; \mathrm{p}<.01)$ and congruent trials $(\mathrm{t}(25)$ $=4.6 ; \mathrm{p}<.001$ ) and unpredictable trials elicited a larger negativity than congruent trials $(t(25)=2.2 ; \mathrm{p}<.05)$. Finally, the interaction between predictability and block was not significant $(\mathrm{F}<1)$ revealing that predictability effects were similar across the two blocks (see Figure 6).

Combining both experiments: To further explore the influence of the social context on lexical predictability, a joint analysis considering experiment, block and predictability was conducted in the time-window of interest (300-500ms), both in the anticipatory and integration phases. For simplicity, only the ANOVA considering the average activity of all the electrodes was computed, and only main effects or interactions involving experiment are described. In the anticipation phase, the results showed no effect of experiment, nor an interaction with block, condition or the two factors (all Fs $<1$ ). In both experiments, we obtained exactly the same result, that is, a larger average positivity for the CP-block and a larger effect of predictability in the CPthan in the C-block.

In the integration phase, no overall differences were observed between experiments $(\mathrm{F}<1)$. The three-way interaction between Experiment, block and condition showed a trend for significance $\left(F(2,94)=2.8, p=.06, \eta^{2}=.05\right)$, consistent with the description of a lexical predictability effect in both blocks of the joint 
Running head: Lexical prediction in conversation

experiment (C-block: $F(2,46)=5.7, p=.006$; CP-block: $F(2,46)=7.6, p=.001)$, but only in the C-block of the solo experiment (C-block: $\mathrm{F}(2,46)=12.3, \mathrm{p}=.000$; CP-block: $\mathrm{F}(2,46)=2.5, \mathrm{p}=.089)$.

In sum, the results revealed that the involvement of speech production in lexical prediction during the anticipatory phase was not modulated by the presence of an interacting confederate. When comprehension was intermixed with production trials, lexical predictability effects in the anticipatory phase were enhanced, and this effect was independent of whether the participant was speaking alone or to a believed partner. The presence of an interlocutor modulated however, the effect of lexical predictability in the integration phase, with the main difference being the observation of a predictability effect when speaking was intermixed with comprehension in the joint context and its absence in the solo context.

\section{DISCUSSION}

The present study aimed to investigate language in interaction by assessing how lexical prediction is modulated by two basic ingredients of a conversation: a) the interleaving between production and comprehension, and b) the presence of an interlocutor. In two experiments tapping lexico-semantic processes during language comprehension, we explored modulations of the N400 component, as an index of lexical prediction, in two distinct phases during language comprehension: before (anticipatory phase) and after auditory comprehension was engaged (integration phase).

Our results revealed that the involvement of speech production in a language task influences lexical prediction processes and the effects vary through the time-course of language comprehension. 
Running head: Lexical prediction in conversation

In the anticipatory phase, lexical prediction, as indexed by the N400 component, was enhanced when comprehension and production trials were intermixed. These results replicate previous findings showing that lexical prediction is encouraged when production is engaged in the task (Ito et al., 2017; Hintz et al., 2016), which provides further evidence for models assuming that prediction is production-based (Lupyan \& Clark, 2015; Pickering et al., 2013) and suggests that similar neurophysiological mechanisms might underlie both processes (Menenti, Pickering, \& Garrod, 2012; Molinaro et al., 2016; Silbert, Honey, Simony, Poeppel, \& Hasson, 2014). In particular, because language production and prediction are top-down processes, that link an intended meaning to the preparation of linguistic forms, neural mechanisms might be similarly engaged in both activities. As such, engaging the production system in the task might have increased the amount of resources devoted to top-down processes, thus facilitating lexical prediction processes during the anticipatory phase of language comprehension (Brothers, Swaab, \& Traxler, 2017).

No influence was observed from the social context on lexical predictability during the anticipatory phase, in contrast. Irrespective of whether participants believed they were interacting with someone or not, the amplitude of the N400 for lexical predictability was increased when the task required switching back and forth from comprehension to production. That is, speaking alone or speaking with a real interactive partner did not modulate lexical predictability at early stages of language comprehension. This result contrasts with previous reports in the joint language literature showing an influence of the interactive partner on lexical processing (e.g., Baus et al., 2014; Kuhlen et al., 2017). While there are important differences between those experiments and ours, one possibility is that the interactive-partner was reputedly in another room in our study, but was physically present in those and this might have 
Running head: Lexical prediction in conversation

affected co-representations of the partner's task (Sebanz \& Knoblich, 2009). Note however that the participants in the current study were all sure that the interlocutor was performing the task along with them.

Regarding integration processes, mixing production and comprehension trials had an impact on lexical predictability during the integration phase and this effect was in turn modulated by the involvement of an interactive partner in the task. In the solo context, where participants were alone doing the task, the N400 effect after target presentation was observed in the comprehension block but not in the comprehension/production block. Color incongruent words (hearing blue after seeing the word SUN) and unpredictable words (hearing BLUE after seeing umbrella) showed a N400 enhancement relative to color congruent words (YELLOW after sun). This effect clearly replicated the influence of lexical prediction during language comprehension (e.g., Lau, Gramfort, Hämäläinen, \& Kuperberg, 2013; Lau \& Nguyen, 2015). Conversely, the effect of lexical predictability diminished when production and comprehension trials were intermixed. These results support Ito et al., (2017) showing that production interferes with integration processes during comprehension (see also Martin et al., 2017). According to the authors, given that production and integration processes do not share mechanisms (top-down vs bottom-up), the engagement of the production system within the task might affect the engagement of integration processes during comprehension (see Huettig, 2015, for a review).

The lack of predictability effects in Martin et al., (2018) was interpreted as that the availability of the production system is necessary for prediction during sentence comprehension. However, in our study, production was fully available during comprehension and nevertheless predictability effects were not obtained during the integration phase. Altogether, these results newly clarify that production influences 
Running head: Lexical prediction in conversation

integration processes regardless of whether production and comprehension are simultaneously (Ito et al., 2017; Martin et al., 2018) or sequentially (Hintz et al., 2016) engaged in the task.

Importantly, as we showed, lexical predictability during the integration phase is modulated by the social context. When participants believed they were interacting with a partner, predictability effects (indexed by the N400) during the integration phase were similar in the comprehension and in the comprehension/production blocks. Thus, the social context proactively influences lexical prediction, which supports the idea that predictions are not automatic but rather strategically generated to facilitate upcoming processing (Brothers et al., 2017; Huettig, 2015). If, as suggested, lexical prediction is costly for the system, comprehenders need to efficiently adapt the amount of resources allocated to prediction according to the relevance of the task at hand (Kuperberg \& Jaeger, 2016; Lupyan \& Clark, 2015). One suggested possibility through which the context might proactively affect language prediction is attention. Within predictive coding models (Friston, 2005; Friston \& Frith, 2015), attention is viewed as a mechanism that can modulate the magnitude of the sensory response and therefore the prediction error signal-i.e., amplifying or reducing it-according to the intention of the listener, the predictability of the stimulus, or the task-demands (see also, Found \& Müller, 1996; Tian \& Poeppel, 2013). Within this framework, the social context, which is assumed to increase attention to others (Klein, Shepherd, \& Platt, 2009), might reduce or amplify sensory responses when relevant. Several studies have demonstrated that social partners (relative to the non-social context) influence communication by boosting attention (Verga \& Kotz, 2013, 2019) or enhancing brain activity (Jeong et al., 2011; Kuhlen \& Abdel Rahman, 2017; Rice \& Redcay, 2016), which in turn influence lexical processing (Baus et al., 2014; Demiral, Gambi, Nieuwland, \& 
Running head: Lexical prediction in conversation

Pickering, 2016) and language learning (Verga \& Kotz, 2013). Our results adhere to those by showing that the attentional gain of social interaction allowed participants overcoming interference from the engagement of prediction-by production mechanisms at later stages of language comprehension (see also Porcu, Bölling, Lappe, \& Liepelt, 2016, for a similar description on joint action performance). That is, the social attention might enhance pre-activation of the lexical representation of color associated to predictable words (yellow), which in turn might enhance prediction error responses when unpredictable or incongruent responses are listened.

\section{$\underline{\text { Conclusion }}$}

In two experiments, we observed evidence that conversational aspects influence the time-course of lexical prediction during auditory language comprehension. The task-wide involvement of production enhances prediction processes during anticipation and this enhancement is not modulated by the social context, as manipulated here. Conversely, the interleaving of production and comprehension reduces integration processes only when participants speak in isolation but not when speaking with a partner, suggesting that language processing is modulated by the interactive context in which conversations occur. Our study is important in revealing how the important social nature of language, particularly the basic ingredients of a conversation, influence lexical processing. 
Running head: Lexical prediction in conversation

\section{ACKNOWLEDGMENTS}

This work was supported by postdoctoral grants to Cristina Baus from the people programme (Marie Curie Actions) of the European Union’s Seventh Framework Programme under REA agreement $n^{\circ} 623845$. 
Running head: Lexical prediction in conversation

\section{REFERENCES}

Baus, C., Sebanz, N., Fuente, V. D. L., Branzi, F. M., Martin, C., \& Costa, A. (2014).

On predicting others' words: Electrophysiological evidence of prediction in speech production. Cognition, 133(2).

https://doi.org/10.1016/j.cognition.2014.07.006

Borovsky, A., Elman, J. L., \& Fernald, A. (2012). Knowing a lot for one’s age: Vocabulary skill and not age is associated with anticipatory incremental sentence interpretation in children and adults. Journal of Experimental Child Psychology, 112(4), 417-436. https://doi.org/10.1016/J.JECP.2012.01.005

Bramão, I., Faísca, L., Forkstam, C., Inácio, F., Araújo, S., Petersson, K. M., \& Reis, A. (2012). The interaction between surface color and color knowledge: Behavioral and electrophysiological evidence. Brain and Cognition, 78(1), 2837. https://doi.org/10.1016/J.BANDC.2011.10.004

Brothers, T., Swaab, T. Y., \& Traxler, M. J. (2017). Goals and strategies influence lexical prediction during sentence comprehension. Journal of Memory and Language, 93, 203-216. https://doi.org/10.1016/J.JML.2016.10.002

Clark, H. H., \& Carlson, T. B. (1982). Hearers and speech acts. Language, 58(2), 332-373. https://doi.org/10.1353/lan.1982.0042

Curran, T., Tucker, D. M., Kutas, M., \& Posner, M. I. (1993). Topography of the N400: brain electrical activity reflecting semantic expectancy. Electroencephalography and Clinical Neurophysiology/Evoked Potentials Section, 88(3), 188-209. https://doi.org/10.1016/0168-5597(93)90004-9

Dell, G. S., \& Chang, F. (2013). The P-chain: relating sentence production and its disorders to comprehension and acquisition. Philosophical Transactions of the Royal Society of London B: Biological Sciences, 369(1634). 
Running head: Lexical prediction in conversation

Demiral, Ş. B., Gambi, C., Nieuwland, M. S., \& Pickering, M. J. (2016). Neural correlates of verbal joint action: ERPs reveal common perception and action systems in a shared-Stroop task. Brain Research, 1649, 79-89. https://doi.org/10.1016/j.brainres.2016.08.025

Dikker, S., \& Pylkkänen, L. (2013). Predicting language: MEG evidence for lexical preactivation. Brain and Language, 127(1), 55-64. https://doi.org/10.1016/j.bandl.2012.08.004

Dikker, S., Silbert, L. J., Hasson, U., \& Zevin, J. D. (2014). On the same wavelength: predictable language enhances speaker-listener brain-to-brain synchrony in posterior superior temporal gyrus. The Journal of Neuroscience : The Official Journal of the Society for Neuroscience, 34(18), 6267-6272. https://doi.org/10.1523/JNEUROSCI.3796-13.2014

Drake, E., \& Corley, M. (2015). Effects in production of word pre-activation during listening: Are listener-generated predictions specified at a speech-sound level? Memory \& Cognition, 43(1), 111-120. https://doi.org/10.3758/s13421-014$0451-9$

Found, A., \& Müller, H. J. (1996). Searching for unknown feature targets on more than one dimension: Investigating a “dimension-weighting” account. Perception \& Psychophysics, 58(1), 88-101. https://doi.org/10.3758/BF03205479

Friston, K. (2005). A theory of cortical responses. Philosophical Transactions of the Royal Society of London B: Biological Sciences, 360(1456).

Friston, K. J., \& Frith, C. D. (2015). Active inference, communication and hermeneutics. Cortex, 68, 129-143. https://doi.org/10.1016/j.cortex.2015.03.025

Gambi, C., Van de Cavey, J., \& Pickering, M. J. (2015). Interference in joint picture naming. Journal of Experimental Psychology: Learning, Memory, and 
Running head: Lexical prediction in conversation

Cognition, 41(1), 1-21. https://doi.org/10.1037/a0037438

García, A. M., \& Ibáñez, A. (2014). Two-Person Neuroscience and Naturalistic Social Communication: The Role of Language and Linguistic Variables in BrainCoupling Research. Frontiers in Psychiatry, 5, 124. https://doi.org/10.3389/fpsyt.2014.00124

Garrod, S., \& Pickering, M. J. (2004). Why is conversation so easy? Trends in Cognitive Sciences, 8(1), 8-11. https://doi.org/10.1016/j.tics.2003.10.016

Hintz, F., Meyer, A. S., \& Huettig, F. (2016). Encouraging prediction during production facilitates subsequent comprehension: Evidence from interleaved object naming in sentence context and sentence reading. The Quarterly Journal of Experimental Psychology, 69(6), 1056-1063. https://doi.org/10.1080/17470218.2015.1131309

Huettig, F. (2015). Four central questions about prediction in language processing. Brain Research, 1626, 118-135. https://doi.org/10.1016/j.brainres.2015.02.014

Ito, A., Corley, M., Pickering, M. J., Martin, A. E., \& Nieuwland, M. S. (2016). Predicting form and meaning: Evidence from brain potentials. Journal of Memory and Language, 86, 157-171. https://doi.org/10.1016/J.JML.2015.10.007 Ito, A., Pickering, M. J., \& Dunn III, M. S. (2017). Effects of language production on prediction: Word vs. picture visual world study. Conference paper at Mental Achitecture for Processing and Learning (MAPLL).

Jeong, H., Hashizume, H., Sugiura, M., Sassa, Y., Yokoyama, S., Shiozaki, S., \& Kawashima, R. (2011). Testing Second Language Oral Proficiency in Direct and Semidirect Settings: A Social-Cognitive Neuroscience Perspective. Language Learning, 61(3), 675-699. https://doi.org/10.1111/j.1467-9922.2011.00635.x Jouravlev, O., Schwartz, R., Ayyash, D., Mineroff, Z., Gibson, E., \& Fedorenko, E. 
Running head: Lexical prediction in conversation

(2019). Tracking Colisteners’ Knowledge States During Language

Comprehension. Psychological Science, 30(1), 3-19.

https://doi.org/10.1177/0956797618807674

Klein, J. T., Shepherd, S. V., \& Platt, M. L. (2009). Social Attention and the Brain. Current Biology, 19(20), R958-R962.

https://doi.org/10.1016/J.CUB.2009.08.010

Krauss, R. M., \& Bricker, P. D. (1967). Effects of Transmission Delay and Access Delay on the Efficiency of Verbal Communication. The Journal of the Acoustical Society of America, 41(2), 286. https://doi.org/10.1121/1.1910338

Kuhlen, A. K., \& Abdel Rahman, R. (2017). Having a task partner affects lexical retrieval: Spoken word production in shared task settings. Cognition, 166, 94106. https://doi.org/10.1016/J.COGNITION.2017.05.024

Kuperberg, G. R., \& Jaeger, T. F. (2016). What do we mean by prediction in language comprehension? Language, Cognition and Neuroscience, 31(1), 32-59. https://doi.org/10.1080/23273798.2015.1102299

Kutas, M., \& Federmeier, K. D. (2011). Thirty Years and Counting: Finding Meaning in the N400 Component of the Event-Related Brain Potential (ERP). Annual Review of Psychology, 62(1), 621-647. https://doi.org/10.1146/annurev.psych.093008.131123

Lau, E. F., Gramfort, A., Hämäläinen, M. S., \& Kuperberg, G. R. (2013). Automatic semantic facilitation in anterior temporal cortex revealed through multimodal neuroimaging. The Journal of Neuroscience : The Official Journal of the Society for Neuroscience, 33(43), 17174-17181.

https://doi.org/10.1523/JNEUROSCI.1018-13.2013

Lau, E. F., Holcomb, P. J., \& Kuperberg, G. R. (2013). Dissociating N400 Effects of 
Running head: Lexical prediction in conversation

Prediction from Association in Single-word Contexts. Journal of Cognitive Neuroscience, 25(3), 484-502. https://doi.org/10.1162/jocn_a_00328

Lau, E. F., \& Nguyen, E. (2015). The role of temporal predictability in semantic expectation: An MEG investigation. Cortex, 68, 8-19.

https://doi.org/10.1016/J.CORTEX.2015.02.022

Léger, L., \& Chauvet, E. (2015). When canary primes yellow: effects of semantic memory on overt attention. Psychonomic Bulletin \& Review, 22(1), 200-205. https://doi.org/10.3758/s13423-014-0657-7

Lupyan, G., \& Clark, A. (2015). Words and the world: Predictive coding and the language-perception-cognition interface. Current Directions in Psychology, 110. https://doi.org/10.1177/0963721415570732

Magyari, L., \& de Ruiter, J. P. (2012). Prediction of Turn-Ends Based on Anticipation of Upcoming Words. Frontiers in Psychology, 3, 376. https://doi.org/10.3389/fpsyg.2012.00376

Mani, N., Daum, M. M., \& Huettig, F. (2016). "Proactive” in many ways: Developmental evidence for a dynamic pluralistic approach to prediction. Quarterly Journal of Experimental Psychology, 69(11), 2189-2201. https://doi.org/10.1080/17470218.2015.1111395

Mani, N., \& Huettig, F. (2012). Prediction during language processing is a piece of cake—But only for skilled producers. Journal of Experimental Psychology: Human Perception and Performance, 38(4), 843-847. https://doi.org/10.1037/a0029284

Martin, C. D., Branzi, F. M., \& Bar, M. (2018). Prediction is Production: The missing link between language production and comprehension. Scientific Reports, 8(1), 1079. https://doi.org/10.1038/s41598-018-19499-4 
Running head: Lexical prediction in conversation

Mantegna, F., Hintz, F., Ostarek, M., Alday P.M., Huettig, F. (2019). Distinguishing integration and prediction accounts of ERP N400 modulations in language processing through experimental design. Neuropsychologia,

\section{https://doi.org/10.1016/j.neuropsychologia.2019.107199}

Menenti, L., Pickering, M. J., \& Garrod, S. C. (2012). Toward a neural basis of interactive alignment in conversation. Frontiers in Human Neuroscience, 6, 185. https://doi.org/10.3389/fnhum.2012.00185

Molinaro, N., Monsalve, I. F., \& Lizarazu, M. (2016). Is there a common oscillatory brain mechanism for producing and predicting language? Language, Cognition and Neuroscience, 31(1), 145-158.

https://doi.org/10.1080/23273798.2015.1077978

Nieuwland, M. S., et al., (2019). Dissociable effects of prediction and integration during language comprehension: Evidence from a large-scale study using brain potentials. Proceedings of the Royal Society B: Biological Sciences.

Pickering, M. J., \& Garrod, S. (2007). Do people use language production to make predictions during comprehension? Trends in Cognitive Sciences, 11(3), 105110. https://doi.org/10.1016/j.tics.2006.12.002

Pickering, M. J., \& Garrod, S. (2013). An integrated theory of language production and comprehension. Behavioral and Brain Sciences, 36(04), 329-347. https://doi.org/10.1017/S0140525X12001495

Porcu, E., Bölling, L., Lappe, M., \& Liepelt, R. (2016). Pointing out mechanisms underlying joint action. Attention, Perception, \&. Retrieved from http://link.springer.com/article/10.3758/s13414-016-1093-8

Redmann, A., FitzPatrick, I., Hellwig, F., \& Indefrey, P. (2014). The use of conceptual components in language production: an ERP study. Frontiers in 
Running head: Lexical prediction in conversation

Psychology, 5, 363. https://doi.org/10.3389/fpsyg.2014.00363

Rice, K., \& Redcay, E. (2016). Interaction matters: A perceived social partner alters the neural processing of human speech. NeuroImage, 129, 480-488. https://doi.org/10.1016/J.NEUROIMAGE.2015.11.041

Rueschemeyer, S.-A., Gardner, T., \& Stoner, C. (2015). The Social N400 effect: how the presence of other listeners affects language comprehension. Psychonomic Bulletin \& Review, 22(1), 128-134. https://doi.org/10.3758/s13423-014-0654-X

Ruiter, J.-P. de, Mitterer, H., \& Enfield, N. J. (2006). Projecting the End of a Speaker’s Turn: A Cognitive Cornerstone of Conversation. Language, 82(3), 515-535. https://doi.org/10.1353/lan.2006.0130

Sebanz, N., \& Knoblich, G. (2009). Prediction in joint action: What, when, and where. Topics in Cognitive Science, 1(2), 353-367.

Silbert, L. J., Honey, C. J., Simony, E., Poeppel, D., \& Hasson, U. (2014). Coupled neural systems underlie the production and comprehension of naturalistic narrative speech. Proceedings of the National Academy of Sciences, 111(43), E4687-E4696.

Stivers, T., Enfield, N. J., Brown, P., Englert, C., Hayashi, M., Heinemann, T., ... Levinson, S. C. (2009). Universals and cultural variation in turn-taking in conversation. Proceedings of the National Academy of Sciences of the United States of America, 106(26), 10587-10592. https://doi.org/10.1073/pnas.0903616106

Tadel, F., Baillet, S., Mosher, J. C., Pantazis, D., \& Leahy, R. M. (2011). Brainstorm: A User-Friendly Application for MEG/EEG Analysis. Computational Intelligence and Neuroscience, 2011, 13. https://doi.org/10.1155/2011/879716 Tanaka, J. W., \& Presnell, L. M. (1999). Color diagnosticity in object recognition. 
Running head: Lexical prediction in conversation

Perception \& Psychophysics, 61(6), 1140-1153.

https://doi.org/10.3758/BF03207619

Tian, X., \& Poeppel, D. (2013). The Effect of Imagination on Stimulation: The Functional Specificity of Efference Copies in Speech Processing. Journal of Cognitive Neuroscience, 25(7), 1020-1036.

https://doi.org/10.1162/jocn_a_00381

Verga, L., \& Kotz, S. A. (2013). How relevant is social interaction in second language learning? Frontiers in Human Neuroscience, 7, 550.

https://doi.org/10.3389/fnhum.2013.00550

Verga, L., \& Kotz, S. A. (2019). Putting language back into ecological communication contexts. Language, Cognition and Neuroscience, 34(4), 536544. https://doi.org/10.1080/23273798.2018.1506886

Wenke, D., Atmaca, S., Holländer, A., Liepelt, R., Baess, P., \& Prinz, W. (2011). What is Shared in Joint Action? Issues of Co-representation, Response Conflict, and Agent Identification. Review of Philosophy and Psychology, 2(2), 147-172. https://doi.org/10.1007/s13164-011-0057-0

Westley, A., Kohút, Z., \& Rueschemeyer, S.-A. (2017). “I know something you don’t know”: Discourse and social context effects on the N400 in adolescents. Journal of Experimental Child Psychology, 164, 45-54. https://doi.org/10.1016/J.JECP.2017.06.016

Yee, E., Ahmed, S. Z., \& Thompson-Schill, S. L. (2012). Colorless Green Ideas (Can) Prime Furiously. Psychological Science, 23(4), 364-369. https://doi.org/10.1177/0956797611430691 
Running head: Lexical prediction in conversation

\section{Footnotes}

1. $F_{\text {corr }}$ and $\mathrm{p}_{\text {corr }}$ determine the threshold of significance. All electrodes showing a significant effect had a F-value above the $\mathrm{F}_{\text {corr }}$ value and a p-value below $\mathrm{P}_{\mathrm{corr}}$. 